\title{
Erratum to: Integrating farm with bio-based practices for food security and sustainability
}

\author{
Sourendra Nath Das 1 - Debabrata Giri • \\ Manas Ghosh
}

Published online: 1 September 2015

(C) Indian Institute of Management Calcutta 2015

\section{Erratum to: Decision (June 2015) 42(2):229-241 \\ DOI 10.1007/s40622-015-0094-0}

In the original publication of the article, the annexure has some Bengali text which is not properly appearing due to font incompatibility. Additionally, Figures 2, 3, 4 , and 5 have text in Bengali script. The correct text of the annexure and corresponding figures with English text are found below:

The online version of the original article can be found under doi:10.1007/s40622-015-0094-0.

S. N. Das $(\bowtie) \cdot$ D. Giri - M. Ghosh Agricultural Training Centre/State Agricultural Management and Extension Training Institute, Ramakrishna Mission Ashrama, Narendrapur, Kolkata, West Bengal 700 103, India e-mail: sourenks@gmail.com URL: http://www.sametiwb.org

D. Giri

e-mail: giriatc@gmail.com URL: http://www.sametiwb.org

M. Ghosh

e-mail: atcrkmnd@gmail.com

URL: http://www.sametiwb.org 


\section{Annex}

Bengali

১) জমিতে চামের যাবতীয় জমা-খরচ এর হিসেব কি লিখে রাখেন ? - হ্যা / না

২)সারা বছরের শষ্য পর্যায় করেছেন কি ? - ই্যা / না

৩) মাটি পরীক্কার ভিত্তিতে জমিতে সার দেন কি ? - ই্যা / না

8) মাটিতে জৈবসার / জীবানু সার / সবুজ সার ব্যবহার করেন কি ? - হ্যা / না

৫) চামের সময় বীজ/ চারা শোধন করেন কি ? - ই্যা / না

৬) জমিতে যখন কোন চাষ হয় না - তখন জমি উন্টে রোদ খাওয়ান কি ? - হ্যা / না

৭) পোকা-মাকড়/রোগের জন্য জৈব ওষুধ ব্যবহার করেন কি ? - হ্যা / না

৮-) তরল জৈবসার তৈরী করতে জানেন কি ? - হ্যা / না

৯) পয়োজনের অতিরিক্ত জলসেচের ফনে কি রোগ-পোকার উপদ্রব বাড়ে কি ? - ই্যা / না ১০)ফসলে শুধুমাত্র সবুজ। নীল সতর্কতা যুক্ত রাসায়নিক কীটনাশক বাবহার করেন কি ? - হ্যা / না

Fig. 2 Year round rainfall pattern of Kamalakantapur village

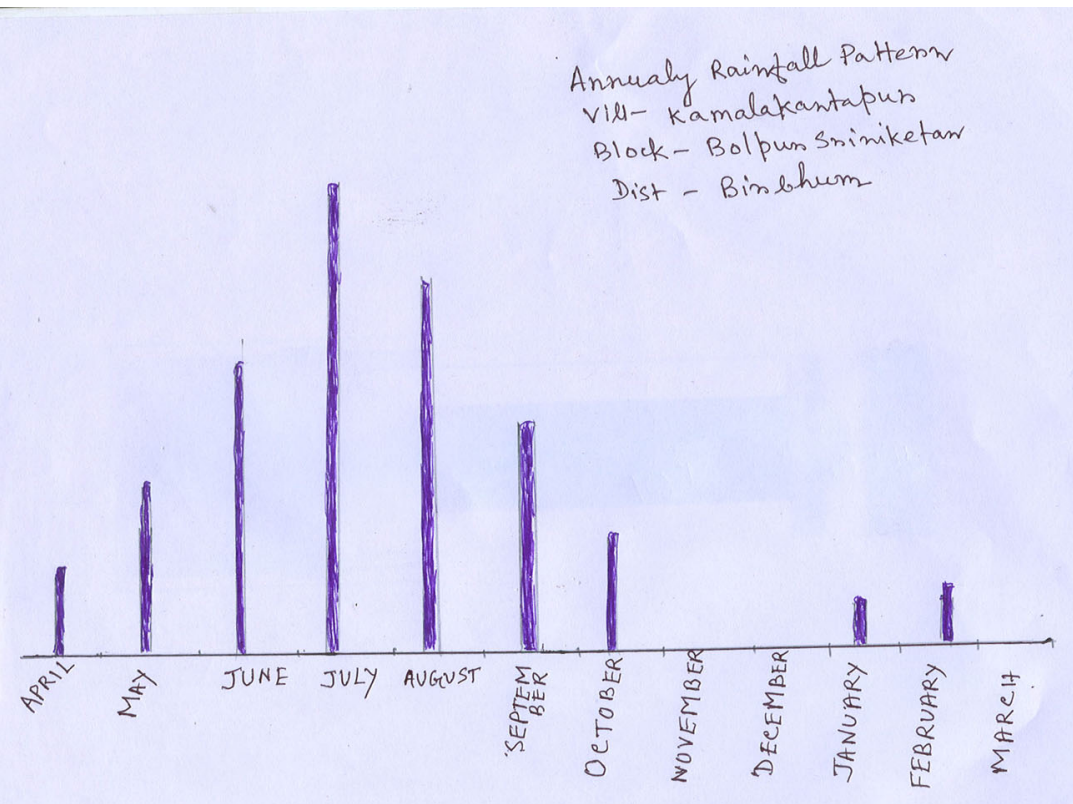


VILL-KAMALAKANTAPUR/BLOCK-BOLPUR SRINIKETAN/DIST-BIRBHUM

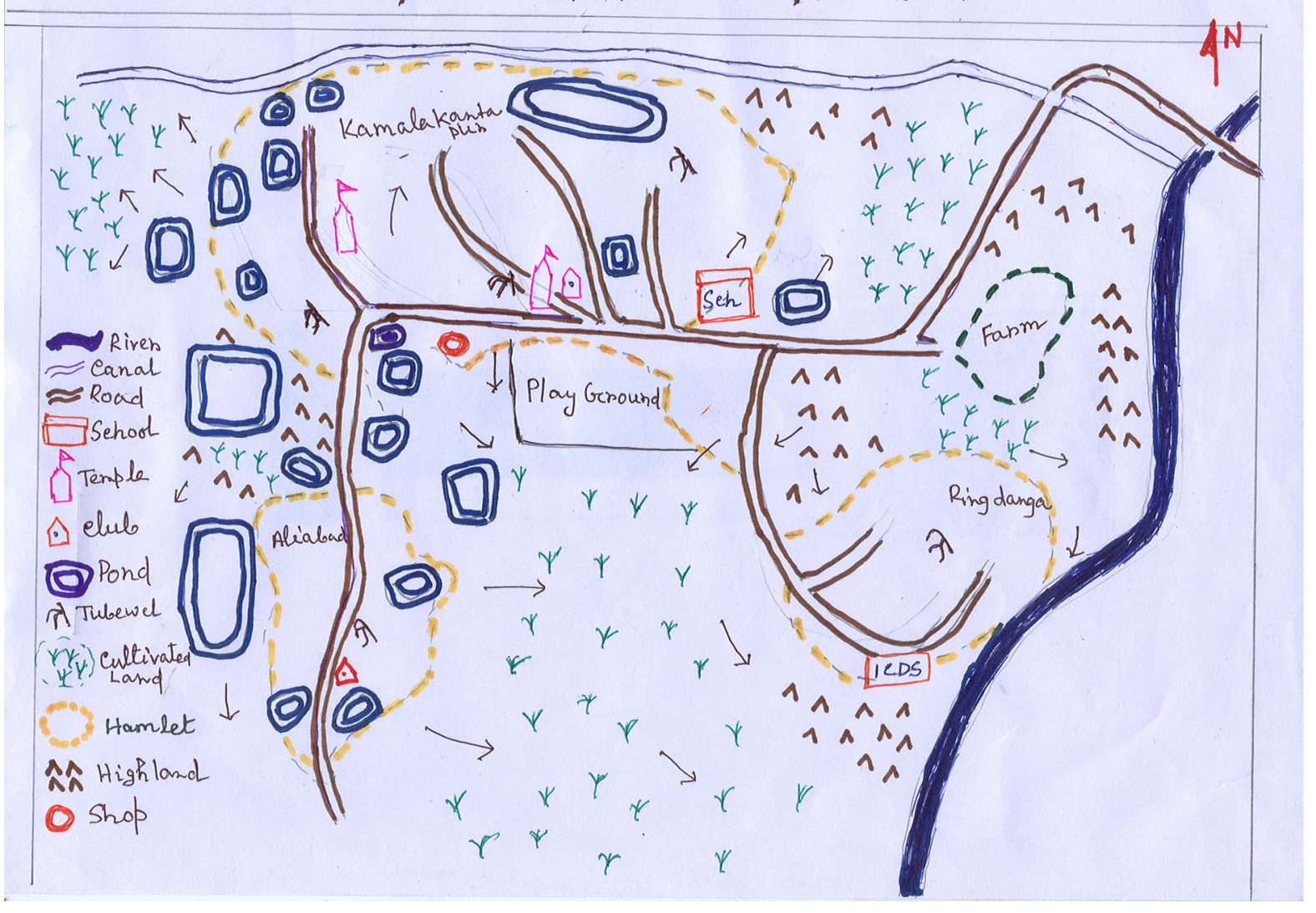

Fig. 3 Resource map of Kamalakantapur village 
Fig. 4 Year round crop planning of farmers of Kamalakantapur village

Fig. 5 Year round kitchen garden planning of farmers of Kamalakantapur village
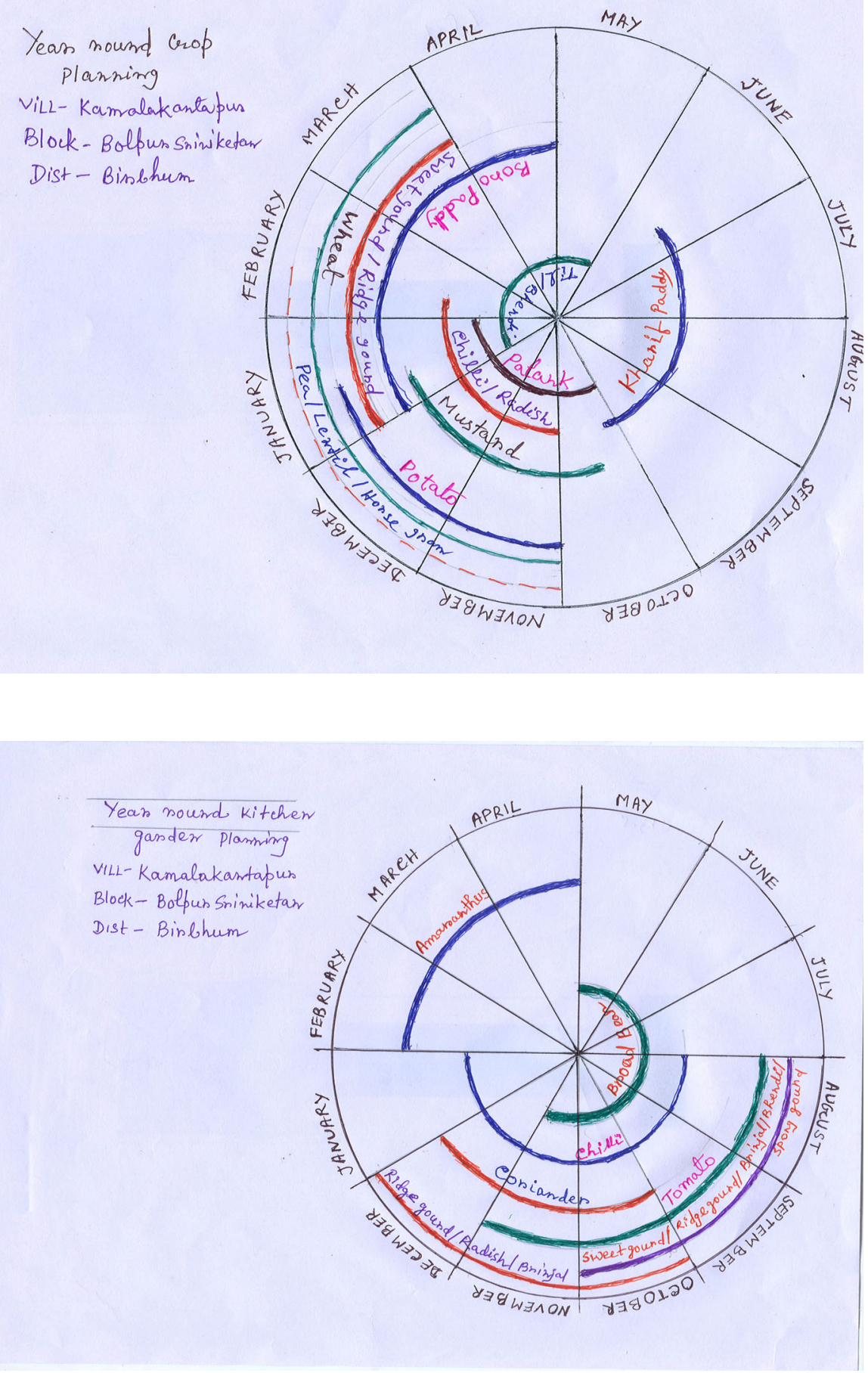